\title{
What do cancer patients' relatives think about addressing cancer family history and performing genetic testing in palliative care?
}

\author{
Jude E. Cléophat ${ }^{1,2,3}$ - Ana Marin ${ }^{4,5,6}$. Sylvie Pelletier ${ }^{1}$ - Yann Joly $\mathbb{D}^{7} \cdot$ Pierre Gagnon $^{1,3,8} \cdot$ Alberte Déry $^{9}$. \\ Jocelyne Chiquette ${ }^{1,3,5,10} \cdot$ Bruno Gagnon $^{1,3,10}$ - Louis Roy ${ }^{5}$. Vasiliki Bitzas ${ }^{11} \cdot$ Hermann Nabi ${ }^{1,3,10,12}$. \\ Michel Dorval ${ }^{1,2,3,13}$
}

Received: 20 May 2019 / Revised: 19 August 2019 / Accepted: 29 August 2019 / Published online: 16 September 2019

(c) The Author(s), under exclusive licence to European Society of Human Genetics 2019

\begin{abstract}
Palliative care may be an opportunity to discuss cancer family history and familial cancer risks with patients' relatives. It may also represent the last opportunity to collect, from dying patients, clinical data and biospecimens that will inform cancer risk assessment and prevention in their surviving relatives. This study aims to explore the perspectives of cancer patients' relatives about cancer heritability, addressing cancer family history, and performing genetic testing in palliative care settings. Thirteen first-degree relatives of cancer patients who died in palliative care participated in the study. Two focus groups were conducted and transcribed verbatim. Two independent coders conducted a thematic content analysis. The themes included: (1) Knowledge of cancer heritability; (2) Experiences and expectations regarding cancer family history discussions, and (3) Views on genetic testing in palliative care patients and DNA biobanking. Participants seemed aware that cancer family history is a potential risk factor for developing the disease. They considered the palliative care period an inappropriate moment to discuss cancer heritability. They also did not consider palliative care providers as appropriate resources to consult for such matters as they are not specialized in this field. Participants welcomed DNA biobanking and genetic testing conducted at the palliative care patients' request. Cancer occurrence within families raises concerns among relatives about cancer heritability, but the palliative care period is not considered the most appropriate moment to address this issue. However, discussions about the risk to cancer patients' relatives might need to be considered on a case-by-case basis.
\end{abstract}

Supplementary information The online version of this article (https:// doi.org/10.1038/s41431-019-0505-3) contains supplementary material, which is available to authorized users.

Michel Dorval

michel.dorval@crchudequebec.ulaval.ca

1 CHU de Québec-Laval University Research Center, Oncology Division, Quebec City, QC, Canada

2 Faculty of Pharmacy, Laval University, Quebec City, QC, Canada

3 Laval University Cancer Research Center, Quebec City, QC, Canada

4 Faculty of Medicine and Health Sciences, Sherbrooke University, Sherbrooke, QC, Canada

5 CHU de Québec-Laval University, Quebec City, QC, Canada

6 CISSS de Chaudière-Appalaches, Ste-Marie, QC, Canada

7 Center of Genomics and Policy, McGill University, Montreal, QC, Canada

\section{Introduction}

Cancer family history is associated with an increased risk of developing the disease [1-3]. Previous studies have reported on cancer family history discussions taking place

8 Department of Psychiatry and Neuroscience, Faculty of Medicine, Laval University, Quebec City, QC, Canada

9 Quebec Palliative Care Association, Granby, QC, Canada

10 Department of Family Medicine and Emergency Medicine, Faculty of Medicine, Laval University, Quebec City, QC, Canada

11 Jewish General Hospital, Palliative Care Unit, Montreal, QC, Canada

12 French National Institute of Health and Medical Research Institute, Epidemiology and Population Health Research Center, INSERM U.1018, Villejuif, France

13 CISSS de Chaudière-Appalaches Research Center, Lévis, QC, Canada 
between patients, relatives, and palliative care providers [4-6]. During these discussions, palliative care providers may have to address patients' and relatives' concerns regarding their cancer family history and related risks for relatives. Such discussions can allow identifying relatives at increased risk of cancer [7] who can be offered genetic testing [8] and risk-reducing strategies [9] if needed. Data from the literature are inconsistent about relatives' interest in discussing cancer genetics and family history in palliative care $[5,10]$. Palliative care providers have voiced concerns about the potential adverse psychosocial effects of engaging families in such discussions during this time [5, 11-13]. Thus, it remains unclear whether cancer patients' relatives would be inclined to discuss their cancer family history in palliative care settings. We found no study reporting opinions and experiences of relatives about having such discussions. The present study aims to gather the perceptions of cancer patients' relatives about cancer heritability, addressing cancer family history, and performing genetic testing in palliative care settings.

\section{Subjects and methods}

\section{Participants}

Eligible for this study were first-degree blood relatives of cancer patients who died at 70 years of age or younger in a palliative care setting. Participants had to be 18 or older and have been in contact with palliative care providers. Patient's death had to occur $>4$ months ago. There was no restriction on the deceased patients' cancer type, except for lung cancer for which the hereditary component is considered less important. We used two recruitment strategies. First, we identified potential participants through bereavement follow-up lists from the palliative care unit of the Hôpital de l'Enfant-Jésus in Quebec City (Quebec, Canada) and a community non-profit organization (Palli-Aide) offering support services to palliative care patients and families in Saguenay (Quebec, Canada). Potential participants were sent an invitation letter or contacted by telephone. The invitation letter contained a stamped envelope and a response card that interested relatives had to complete and mail back. As few potential participants showed interest in the study, we used a second strategy. On 24th May, 2017, we sent the invitation letter to employees at the $C H U$ de Québec-Université Laval (Quebec City University Hospital-Laval University) through an electronic mailing list. Individuals who replied to these invitations were then contacted by phone by the research coordinator (SP) who presented the study and screened potential participants for eligibility. A letter confirming study participation and two copies of the study information and consent form were sent to prospective participants who met the inclusion criteria.

\section{Data collection}

Two focus group sessions were held in Quebec City (Canada) in June 2017. An independent, experienced, female professional moderator from a private research firm conducted the group discussions in a room equipped for this purpose. A semi-structured discussion guide (Supplementary Document 1), developed by the research team, was used to engage discussions with participants on their (1) knowledge of cancer heritability and experiences with cancer within their family and (2) experiences and expectations regarding discussing cancer family history in palliative care settings. In addition, a fictitious case of a palliative care cancer patient asking for genetic testing was used as a prompt to gather perspectives on biological sampling in palliative care for genetic testing or storage. The group discussions lasted 90 and $120 \mathrm{~min}$, respectively. Each participant received $\$ 70$ (CAD) compensation. The focus groups were digitally audio and video recorded, and discussions were transcribed verbatim by trained firm employees.

\section{Data analysis}

A thematic analysis of the focus groups transcripts was conducted. We built an initial codification framework based on the themes in the discussion guide and study objective, which was to explore participants' perceptions about cancer heritability, addressing cancer family history, and performing genetic testing in palliative care settings. Each theme and subtheme was given a numerical code. Two research team members (AM and JEC) independently read and coded the transcripts according to the analysis framework. Using an inductive approach [14], they supplemented the initial framework with emergent subthemes. Codification differences were resolved by consensus. Coded text excerpts were tabulated under their corresponding theme and subtheme. Polysemic excerpts were placed under several subthemes. Findings from these excerpts were abstracted.

\section{Results}

\section{Participants}

Eighty-two potentially eligible participants were identified through the bereavment lists (first recruitment strategy) among whom six replied to the invitation. Twenty-eight responded to the electronic invitation mail. A total of 34 
cancer patients' relatives expressed interest in participating in the study Of these, 17 were subsequently excluded: three were the spouse/partner of the deceased relative, two were not available on the focus group dates, two had a relative deceased at $>70$ years of age, one had not been in contact with the palliative care team, one had a relative who passed away $<4$ months ago, one had a relative who died $>25$ years ago, three had a relative who died from lung cancer, and four were not reachable. Thus, 17 relatives were found to be eligible. One withdrew from the study for medical reasons and three did not show up to the focus groups. Finally, five and eight relatives participated in the first and second focus group, respectively. They were mostly women (92\%) and aged 30 to 57 years (Table 1). Most were either employed as health care professionals $(54 \%)$ or employees in a health care institution (31\%). Cancer sites among first-degree and more distant relatives were diverse (Supplementary

Table 1 Characteristics of participants

\begin{tabular}{|c|c|c|c|}
\hline & $\begin{array}{l}\text { First } \\
\text { focus group }\end{array}$ & $\begin{array}{l}\text { Second } \\
\text { focus group }\end{array}$ & Total \\
\hline Number of participants & 5 & 8 & 13 \\
\hline Age (range) & $30-37$ & $32-57$ & \\
\hline \multicolumn{4}{|l|}{ Gender } \\
\hline Male & 1 & - & 1 \\
\hline Female & 4 & 8 & 12 \\
\hline \multicolumn{4}{|l|}{$\begin{array}{l}\text { Relationship with the } \\
\text { deceased relative }\end{array}$} \\
\hline Son/daughter & 5 & 7 & 12 \\
\hline Mother/father & - & 1 & 1 \\
\hline \multicolumn{4}{|l|}{$\begin{array}{l}\text { Age of death of the } \\
\text { affected relative (years) }\end{array}$} \\
\hline$<60$ & 1 & 6 & 7 \\
\hline $60-65$ & 3 & 2 & 5 \\
\hline Unspecified & 1 & - & 1 \\
\hline \multicolumn{4}{|l|}{$\begin{array}{l}\text { Deceased relative's } \\
\text { primary cancer site }\end{array}$} \\
\hline Breast & 1 & 0 & 1 \\
\hline Ovaries & 0 & 1 & 1 \\
\hline Colon & 1 & 1 & 2 \\
\hline Stomach & 1 & 0 & 1 \\
\hline Leukemia & 1 & 0 & 1 \\
\hline $\begin{array}{l}\text { Non-Hodgkin } \\
\text { lymphoma }\end{array}$ & 1 & 2 & 3 \\
\hline Brain & 0 & 1 & 1 \\
\hline Bone & 0 & 2 & 2 \\
\hline Neuroblastoma & 0 & 1 & 1 \\
\hline \multicolumn{4}{|l|}{$\begin{array}{l}\text { Time elapsed since the } \\
\text { affected relative's death }\end{array}$} \\
\hline$\leq 5$ years & 4 & 2 & 6 \\
\hline$>5$ years & 1 & 6 & 7 \\
\hline
\end{tabular}

Table 1). Four participants had a first-degree relative who died from breast, colon, or ovarian cancer.

\section{Theme 1: Knowledge of cancer heritability and experiences with cancer in families}

Relevant quotes pertaining to this theme are provided in Table 2.

\section{Subtheme 1.1: Knowledge of cancer heritability}

Most participants were aware of the hereditary component of cancers. They associated their cancer family history with an increased risk of developing the disease for themselves and their children (Quotes 1 and 2). Breast and colon cancer heritability was considered well-known in the general population. (Quote 3). Some participants revealed that they became aware of the hereditary aspect of cancers through (a) their profession, (b) information provided by their entourage, or (c) their affected relative urging them to "get screened" (Quote 4). Still, some participants perceived their late relative's cancer as "coming out of nowhere" or resulting from environmental factors.

\section{Subtheme 1.2: Family member concerns}

Most participants reported that learning of a family member's cancer diagnosis and related death aroused fears and worries (Quote 5). Some were concerned about transmitting a deleterious genetic variant to their offspring (Quote 6). One even pursued genetic assessment because she did not want her child to be "without her mother". After their family members' death, some participants tended to associate any sign or symptom with cancer (Quote 7).

\section{Subtheme 1.3: Timing for self-questioning}

Most participants reported worrying about their cancer risk mostly after the death of their affected relative (Quote 8). For some, these concerns surfaced years later, when they became parents (Quote 9), or when asked by a physician, aware of their cancer family history, to undergo thorough testing for a seemingly benign medical condition. Participants did not perceive palliative care as the ideal time to worry about their own health as the dying patient's health was deemed more important during this period (Quote 10).

\section{Theme 2: Experiences and expectations regarding cancer family history discussions}

Relevant quotes pertaining to this theme are provided in Table 3. 
Table 2 Quotes related to participants' knowledge of cancer heritability and experiences with cancer in families

Subthemes Selected quotes

Knowledge of cancer heritability

Quote 1: "So, when it happened to my mother, it was ...directly linked to me. My father too. I said to myself: Okay, so now [because of] the genetics...like it or not, you make the connection, and it goes downwards; I won't escape from it." Participant 8

Quote 2: "it became clearer when my mother had her cancer ... the same thing happened to my sister not long after. So we said: ... there must be something. ...we wanted to know [...] if it was genetic or something like that." Participant 1

Quote 3: " people question themselves [...] less regarding familial history [of cancer] when it comes to certain types of cancers [...]. We know a little bit more [...] what to do, [...]. I wasn't told [by providers]: [...], it's not genetic, because it's known." Participant 11

Quote 4:"It came from her [her mother], yes. She was raising awareness [about the risk of cancer] and telling everyone to go get checked because ultimately [...] if it's found in time it's okay, but when you have symptoms, it's often too late [...]" Participant 11

Family member concerns

Quote 5: "I think we automatically worry when there's someone with cancer in the family knowing that there are more and more cancer cases, more and more palliative care cases. I think it worries more and more people." Participant 8

Quote 6: "I mostly experienced guilt because it's my child, and the fear that my next children would have cancer [...] So, at some point during the pregnancy, something happened that caused a mutation (genetic variant)." Participant 10

Quote 7: "Well, sure in my case, my father, it was gastric cancer. So, we inquired [...] to find out about the first symptom, to know if we too were affected by that type of cancer[...] It seems to me that we notice them [cancer symptoms]. I get the feeling that we are more alert near death; we see them [cancer symptoms] where there are none." Participant 2

Timing for self-questioning about Quote 8: "It was after death. Once death occurs, that's when you actually start to think." Participant 2 cancer heritability

Quote 9: " the self-questioning [...] has always been present, but really started, [...] during the [genetic] process [...]. It's pretty recent. [...] Since having my child, I say to myself: it's better to know sooner than later." Participant 13

Quote 10: "I came back to be near my mother, with my family. [...] it lasted for a long time [...] So during that time, I thought about it [her own risk of cancer] more or less. [...] the concern was there, but I didn't have the time to focus on it." Participant 8

\section{Subtheme 2.1: Discussing cancer family history within families}

Some participants reported discussions on cancer heritability, prevention strategies and genetic testing with their affected relative, or other family members. However, participants described cancer patients as reluctant to expand on their health condition (Quotes 11 and 12).

\section{Subtheme 2.2: Addressing cancer family history in palliative and curative care settings}

No participant reported having ever discussed cancer family history with health professionals in palliative care (Quote 13). During the curative phase, some questioned their relative's attending physician about cancer heritability (Quote 14). After their relative's passing, others directed their questions to their own family physician (Quote 15). A few participants felt that, when questioned about cancer heritability, physicians tried reassuring them by stating that there was no risk of hereditary transmission to family members. However, physicians did not always succeed in dispelling worries, and instead, participants thought that physicians had limited knowledge of cancer genetics. Sometimes, physicians' answers left participants unsatisfied and doubtful (Quote 16). Nevertheless, a few participants seemed satisfied with physicians' response to their concerns since they were geared toward genetic testing, urgent or enhanced cancer screening.

\section{Subtheme 2.3: Issues about discussing cancer family history along the cancer care trajectory}

Participants did not perceive palliative care as an appropriate setting for addressing cancer family history (Quote 17). They did not consider palliative care providers as the appropriate resource to consult for such matters as they are not known to be specialized in this field. Owing to the emotional burden brought on by the end of life, and to keep their focus on the dying patients, participants preferred being informed about or discussing cancer heritability after the death of their relative (Quote 18). They identified oncologists and specialized genetic resources as the appropriate professionals with whom they should have such 
Table 3 Quotes related to the experiences and expectations of participants regarding cancer family history discussions in the cancer care trajectory

Subthemes Selected quotes

Discussing cancer family history within families

Quote 11: "but my mother didn't talk much about her cancer. But, we did have a discussion to know what type of cancer she had, to know if she had a mutation (genetic-variant)-related cancer type, which is transmissible. "Participant 3

Quote 12: “he really didn't want us to worry about that; he didn't want us to suffer so much, at least, he wanted us to suffer as little as possible [...]but, I didn't want to worry him either, to have him think: Oh my God, am I going to pass that on to my son?" Participant 4

Addressing cancer family history in palliative and Quote 13: "And, in palliative care, it [cancer family history] was never discussed. I never curative care settings asked the question, and no one talked to me about it either." Participant 4

Quote 14: "My brother and I when we were... it wasn't during palliative care but during the hospitalization. Because, when he [her father] was diagnosed with leukemia, he had to be kept in the hospital for a long time, because he was also being kept to have a transplant. And, basically, that's it, we asked the question [about the hereditary transmission risk] to the hematologist and he said no [there was none]." Participant 5

Quote 15: “Then, following my father's death, I have a family physician who I don't see on a regular basis, I don't have any health issues, I see him maybe once a year on average. And, I told him about it [my family history of cancer]." Participant 4

Quote 16: "That's it, and when I told him [the physician] about it [cancer family history], I kind of got the impression that, not that it was not necessary to talk to him about it, but he didn't start a discussion about it. He said okay, took note of it...but the fact that he didn't seem too worried...left me a little bit unsatisfied... Okay, so he said that, but can I get someone to double-check? Is there a second physician I could call?" Participant 4

Issues about discussing cancer family history along the cancer care trajectory
Quote 17: "I don't think that it would have been the best moment...palliative care is a very emotional moment, it's really not a good time. You are mostly there for the person who is dying." Participant 3

Quote 18: "I think it's important to catch those families when they are in palliative care. Is it the best time to do that? No, but it does take something afterwards, some sort of follow-up, maybe something to raise awareness, but not during palliative care, I don't think it's the best way." Participant 3

Quote 19: "For me, family physicians for sure have a role to play in the follow-up, however, I don't know if it is something that is usually done, but to meet with either an oncologist or someone who knows about that [genetics] following the death [is better]. Because, I'm sorry, but family physicians don't know anything about that [genetics]." Participant 2

Quote 20: "It might actually be good in palliative care... with the psychologist and all that, to say: is that a concern of yours? Just to bring it up maybe, and afterwards, it's up to us to follow-up, take the next steps... it [the palliative period] is so full of emotions and stuff. It is not something we necessarily think about at the time, in palliative care." Participant 3

Quote 21: "as soon as we hear the word cancer, we should discuss the topic [cancer heritability] and not wait until the palliative care stage and death." Participant 8

Quote 22: "That the attending physician takes the time to sit down with the family and discuss it [cancer family history]. If only to discuss the case of the cancer-affected relative and implications all around including genetics." Participant 3

Quote 23: "For people whose disease lasts for six months and progresses rapidly, you have less time to think about that" Participant 3

Quote 24: “I don't think there is a good time or good way. Whether it's a close relative or a physician who faces this issue [cancer heritability] all week long, to bring it up, I think...I really think it's on a case-by-case basis." Participant 8 discussions. (Quote 19). However, for well-known heritable cancers, some participants stated they would appreciate being briefly notified about the risk of hereditary transmission during the palliative care period. They felt that this notification could prompt them to obtain information and appropriate management after the death of their relative. Participants identified several opportunities and strategies that could be used to inform family members about a potential familial cancer predisposition, including casual conversations between palliative care providers and family members, information leaflets in palliative care settings, websites, hotlines, and bereavement follow-up phone calls. Several types of palliative care providers were deemed capable of notifying family members about this potential 
predisposition, including physicians, nurse navigators, liaison nurses, psychologists, and social workers (Quote 20).

Some participants showed interest in receiving information on cancer heritability at the time of diagnosis or during their relative's curative treatments. (Quote 21). For slowgrowing cancers, participants thought that there was enough time for oncologists or attending physicians to discuss cancer family history (Quote 22) but not for rapidly progressing cancers (Quote 23). They felt that palliative care cancer patients themselves were capable of informing family members about potential cancer risks. However, participants expressed concern that cancer patients might feel guilty, uncomfortable, or be unwilling to talk about their disease. Some participants thought that family physicians should routinely ask questions about cancer family history and associated risks. Other participants felt that it was their duty to ask health care providers about their risk of cancer. As a conclusion to discussing cancer family history in care settings and owing to the diverse opinions expressed in this regard, participant 8 thought that there was no perfect moment or way to initiate cancer heritability discussions given that such elements rely ultimately on the preferences of involved relatives and health care providers (Quote 24).
Theme 3: Views on genetic testing in palliative care patients and DNA biobanking

Relevant quotes pertaining to this theme are provided in Table 4.

\section{Subtheme 3.1: Perception of genetic testing in palliative care patients}

Genetic testing in palliative care at the patients' request was welcomed by participants. It was perceived as a gift, a legacy, a legitimate last will from the dying patient that deserves to be honored. They felt that genetic testing should be offered automatically to patients when hereditary cancer is suspected and performed well before palliative care. Participants thought that this test could represent an alternative to cancer family history discussions where issues about the transmission of genetic variants might be raised (Quote 25). They felt that this test could be even more relevant as it might influence their own medical care in the future.

Participants reported wanting to be informed about genetic testing performed during their relatives' palliative care through meetings with their late relative's attending

Table 4 Quotes related to the views of participants on genetic testing in palliative care patients and DNA biobanking

Subthemes Selected quotes

Perceptions of genetic testing in palliative care patients

Perception of DNA sampling for biobanking
Quote 25: "It's just that caregiving staff must inform [family members] that a test was done. I can see it being done in palliative care. You can say: did you know that your mother passed this test, if 1 day you need the results, just know that they are available... yes, even at the hospital [...] As opposed to, did you know that you might be a mutation (genetic variant) carrier which of course will cause a bit of a panic [in family members] [...] In any case, in my opinion, I think it's appropriate." Participant 3

Quote 26: "Perhaps it could also be: would you like us to send the results to your family physician? Maybe it's the family physician that's more suited to analyze the information that is being transmitted. Because sometimes, it may not be as simple as saying: yes, it is inherited. There might be other things that need to be explained." Participant 4

Quote 27: "My only concern is being admitted in palliative care before being able to pass it [the test]. In my mother's case, she received palliative care at home. I don't see how it [the test] could have been done there." Participant 8

Quote 28: "In my father's case, in his last weeks of life, he was not always conscious, even not conscious at all during the last two weeks. Therefore, not that it's too late but, I think it should be, let's say, the oncologist who monitors the patient [who should initiate the genetic testing process]." Participant 4

Quote 29: "If ever biobanks do exist, family physicians should be made aware of them." Participant 11

Quote 30: "Let's say we meet our specialist physician ten years later because of some medical condition. If analyzing this data [biobanks] can help caregivers, especially if it involves information regarding several family members, then, in that case, it will be even more helpful for the medical team." Participant 4

Quote 31: "Yes. We don't know, in five years, in ten years, maybe they are going to discover many other things. (Participant 4 nodded in agreement). And if it can be used for research in the meantime [so be it]" Participant 2

Quote 32: "But, I think on the other hand that, when someone gives a specimen for a test, I don't think it can be used if the consent was not obtained" Participant 5 
physicians, brief notifications in palliative care, letters, phone calls, or by their family physician following their relative's death. Most showed interest in receiving test results after their relative's death, either from a specialized genetics professional or their family physician, and preferably, after obtaining the palliative care patient's consent (Quote 26). However, the ability to disclose deceased patients' medical information to family members was questioned due to confidentiality requirements. The potential impacts of test results on patients' relatives' insurability also raised concerns. Logistic limitations were mentioned as barriers to end-of-life genetic testing such as when the dying patient resides at home or when he/she is unable to make informed decisions (Quotes 27 and 28).

\section{Subtheme 3.2: Perception of DNA sampling for biobanking}

Sampling and storage of genetic material for prospective genetic testing were well perceived by participants. They stressed the need to raise awareness about biobanks among health care providers and the general population (Quote 29). Some participants thought that blood relatives, particularly first-degree relatives, should be informed of the existence of stored DNA samples. They acknowledged that these samples might be used in the medical care of family members (Quote 30). Some participants were aware that these samples might also be used for research purposes (Quote 31). However, obtaining dying patients' consent was a concern expressed during the discussions (Quote 32).

\section{Discussion}

In this study, relatives of cancer patients reported that they never discussed cancer heritability in palliative care. They did not perceive this setting as appropriate for addressing this issue. They did not consider palliative care providers the appropriate professionals to consult on cancer heritability. They would rather discuss this issue with specialized resources during the diagnosis disclosure process, during curative care or after the death of their relative. However, some participants seemed inclined to accept being briefly informed about cancer heritability in palliative care. Providers, relatives, and patients themselves were designated as capable of bringing up the topic. The occurrence of cancer and related death within families raised concerns among participants about familial cancer risk. These concerns surfaced after their relatives' death. In some cases, these concerns were shared with family members, the affected relative while still alive, and physicians. Participants' degree of satisfaction towards the way their concerns were handled by physicians was variable. All participants viewed obtaining genetic samples from palliative care patients for testing or storage as appropriate, informative, and usable for prospective familial medical care and research.

To our knowledge, this is the first study that questioned relatives of deceased cancer patients about addressing cancer family history and genetic testing in palliative care settings. Palliative care providers in previous studies have reported that palliative care is not the preferred setting for discussions about cancer heritability given the psychosocial and medical issues that patients and families must face at that time [5, 11-13]. Consistent with our results, hospice nurses in a previous study considered that, in palliative care, family members' focus is on the dying relative and on being present for him/her during the end of life [11]. They also considered that the bereavement period is a better time for addressing cancer family history. Similar to participants in this study, Quillin et al. [15] emphasized practical considerations and advantages of performing genetic sampling for biobanking in dying cancer patients. However, genetic testing in palliative care cancer patients is not always welcomed by family members as reported by hospice nurses in Metcalfe et al. [13]. They reported witnessing complex grief, familial tensions, and disagreements over genetic testing, as well as guilt, anxiety, fear, anger, hopelessness, stress, denial, and blame.

Divergent opinions from providers, relatives, and patients about the suitability of cancer heritability discussions in palliative care are also reported in the literature. While some palliative care providers $[5,6,11-13]$ and participants in our study appeared reluctant to initiate discussions about cancer family history, most palliative care cancer patients in Abusamaan et al. study [16] wished that cancer heritability and prevention were discussed in the end-of-life context. Interest in receiving genetic services in palliative care was also noted among patients in Quillin et al. [17]. As reported here, cancer patients are sometimes those raising awareness among their relatives concerning a potential familial cancer predisposition and preventive options [18]. Questioning palliative care patients and families about their willingness to discuss cancer family history and providing educational materials (leaflets, booklets, posters, hotlines, and websites) could potentially foster such discussions. Moreover, providers and family members might find it more acceptable and feasible to prompt discussions around cancer family history in palliative care if the dying patient raises the issue and shows interest in discussing it. Further research is necessary to explore this hypothesis.

Findings should be considered in light of study limitations. Firstly, recruitment constraints led us to reach out to participants who are majorly health care professionals or employees in a health care institution. These participants might have a better understanding of cancer genetics and be 
more aware of the risks related to cancer family history than the general population [19]. They could, therefore, be more concerned, more willing to discuss these risks after the death of their relatives, and more welcoming of genetic testing and sampling in palliative care. Eligibility criteria also prevented us from documenting the perceptions of participants with relatives deceased in palliative care uniquely from cancers known for their hereditary component such as breast, ovarian, colon, and prostate cancer. Nonetheless, this allowed us to gather the opinions of relatives who experienced diverse types of cancer, which better reflects the reality of palliative care. Secondly, although focus groups are well suited for exploring topics that are not yet well-understood [20], our small sample size limits the generalizability of the results. Thirdly, although similar opinions were expressed in both focus groups, additional investigations involving participants from diverse backgrounds might provide further insights and potentially contribute to reaching saturation. Fourthly, as most participants' experience in palliative care goes back several years, there is a risk of recall bias in reporting their recollections. Strengths of the study included using a unique analytic framework thereby increasing internal validity, coding and interpreting transcript contents by two different investigators, and reaching consensus on all findings.

Our findings might be of interest to researchers and health professionals involved in cancer prevention. Ideas proposed by relatives can contribute to developing strategies aiming to raise awareness about hereditary cancers among families during palliative care. Palliative care providers' lack of knowledge about hereditary cancers and genetic resources has been reported in the literature $[4,5,11,13,15]$. Despite relatives' preference in this study for not addressing cancer family history at the end of life, palliative care providers may still have to face and discuss issues related to hereditary cancers. Basic training in genetics may allow them to provide adequate answers and better manage cancer patients and family members.

Before discussing hereditary cancers with families, however, palliative care providers should be aware of, and take into account any existing ethical and legal frameworks guiding these discussions. According to an analysis conducted by Saulnier et al. [21], the norms governing these discussions in Belgium, France, and Quebec lack specificity when it comes to communicating genetic information in the end-of-life context. Suitable rules and laws that take into account patients', providers' and families' opinions might be needed for, timely and adequately, addressing cancer heritability around the end of life.

There might be a need to disseminate knowledge on hereditary cancers and genetic resources throughout the whole cancer care trajectory as study participants did not always perceive family and specialist physicians as competent to deal with their concerns regarding their cancer risk. Adapted procedures and validated supporting tools to broach the topic with relatives could be developed. Validated tools for collecting family history and assessing relatives' cancer risk might also be helpful to health care professionals [22-25].

More studies are needed to clarify the perspective of palliative cancer patients, relatives, and health care providers about addressing cancer heritability in a palliative care context. Their perspectives, keeping in mind ethical considerations, may contribute to developing protocols that will help clinicians navigate questions regarding cancer heritability.

\section{Conclusion}

This study sheds light on cancer patient relatives' awareness of the hereditary component of cancers, their experiences, and expectations about addressing cancer family history, genetic testing, and biobanking during and after the end-oflife period. Discussing cancer family history appeared as a case-by-case initiative. Participants proposed different strategies and considered providers, patients, or relatives capable of initiating cancer family history discussions at any point during the cancer care trajectory, including the palliative care setting. Considering the continuous advances in genomic-based medicine, it might be necessary to make genetics-based education available to providers and take ethical frameworks into account if relatives' cancer risk is to be addressed in palliative care.

Acknowledgements We thank Sue-Ling Chang for linguistic editing of the manuscript.

Funding This study was funded by the Fonds de Recherche $d u$ Québec-Santé and the Direction générale de Cancérologie du Québec. JEC is a recipient of scholarships from the Fonds d'Enseignement et de Recherche (Faculty of Pharmacy, Laval University), Laval University Cancer Research Center, Laval University Research Chair in Palliative Care, and the Fonds de Recherche du Québec-Santé. HN holds an Investigator Award (Junior 2) from the Fonds de recherche du Québec-Santé.

\section{Compliance with ethical standards}

Conflict of interest The authors declare that they have no conflict of interest.

Ethical approval This study was approved by the Research Ethics Review Board of the CHU de Québec-Université Laval (Quebec City University Hospital-Laval University). During the recruitment process, participants were provided with information about the study and on their rights and responsibilities. Each participant signed a consent form before the focus group. First names only were used during group discussions to maintain confidentiality. To report participants' quotes, first names were replaced by numbers. Participants were informed that 
research team members (JEC, AM, HN, MD, SP) would be observing the focus group sessions behind a one-way mirror to record field notes.

Publisher's note Springer Nature remains neutral with regard to jurisdictional claims in published maps and institutional affiliations.

\section{References}

1. Pharoah PD, Day NE, Duffy S, Easton DF, Ponder BA. Family history and the risk of breast cancer: a systematic review and meta-analysis. Int J Cancer. 1997;71:800-9.

2. Noe M, Schroy P, Demierre MF, Babayan R, Geller AC. Increased cancer risk for individuals with a family history of prostate cancer, colorectal cancer, and melanoma and their associated screening recommendations and practices. Cancer Causes Control. 2008;19:1-12.

3. Bevier M, Sundquist K, Hemminki K. Risk of breast cancer in families of multiple affected women and men. Breast Cancer Res Treat. 2012;132:723-8.

4. Gonthier C, Pelletier S, Gagnon P, Marin A, Chiquette J, Gagnon $\mathrm{B}$, et al. Issues related to family history of cancer at the end of life: a palliative care providers' survey. Fam Cancer. 2018;17:303-7.

5. Dearing A, Taverner N. Mainstreaming genetics in palliative care: barriers and suggestions for clinical genetic services. J Community Genet. 2018;9:243-56.

6. Cleophat JE, Pelletier S, Joly Y, Gagnon P, Dery A, Marin A, et al. Addressing cancer family history at the end of life: How frequent, relevant, and feasible is it?: a survey of palliative care providers. Palliat Med. 2019;33:856-8.

7. Niendorf KB, Geller MA, Vogel RI, Church TR, Leininger A, Bakke A, et al. A model for patient-direct screening and referral for familial cancer risk. Fam Cancer. 2016;15:707-16.

8. Hampel H, Bennett RL, Buchanan A, Pearlman R, Wiesner GL, Guideline Development Group ACoMG. et al. A practice guideline from the American College of Medical Genetics and Genomics and the National Society of Genetic Counselors: referral indications for cancer predisposition assessment. Genet Med: Off J Am Coll Med Genet. 2015;17:70-87.

9. Hartmann LC, Lindor NM. The role of risk-reducing surgery in hereditary breast and ovarian cancer. New Engl J Med. 2016; 374:454-68.

10. Roeland EJ, Dullea AD, Hagmann CH, Madlensky L. Addressing hereditary cancer risk at the end of life. J Oncol Pract. 2017;13: e851-6.

11. Lillie AK, Clifford C, Metcalfe A. Caring for families with a family history of cancer: why concerns about genetic predisposition are missing from the palliative agenda. Palliat Med. 2011;25:117-24.

12. Ingleby LJ. Clinical genetics in a palliative care setting: a qualitative exploration of the barriers and levers staff report when discussing family risk of BRCA1 and BRCA2 mutations. Leicester: University of Leicester; 2015.

13. Metcalfe A, Pumphrey R, Clifford C. Hospice nurses and genetics: implications for end-of-life care. J Clin Nurs. 2010; 19:192-207.

14. Elo S, Kyngas $\mathrm{H}$. The qualitative content analysis process. J Adv Nurs. 2008;62:107-15.

15. Quillin JM, Bodurtha JN, Siminoff LA, Smith TJ. Physicians' current practices and opportunities for DNA banking of dying patients with cancer. J Oncol Pract. 2011;7:183-7.

16. Abusamaan MS, Quillin JM, Owodunni O, Emidio O, Kang IG, Yu B, et al. The role of palliative medicine in assessing hereditary cancer risk. Am J Hosp Palliat Care. 2018; 35:1490-7.

17. Quillin JM, Emidio O, Ma B, Bailey L, Smith TJ, Kang IG, et al. High-risk palliative care patients' knowledge and attitudes about hereditary cancer testing and DNA banking. J Genet Couns. 2018;27:834-43.

18. Lillie A. The missing discourse:how does the family history of cancer affect the care needs of palliative care patients? Birmingham: University of Birmingham; 2009.

19. Krakow M, Ratcliff CL, Hesse BW, Greenberg-Worisek AJ. Assessing genetic literacy awareness and knowledge gaps in the US population: results from the health information national trends survey. Public Health Genomics. 2017;20:343-8.

20. Kitzinger J. Qualitative research. Introducing focus groups BMJ. 1995;311:299-302.

21. Saulnier KM, Chan B, Cinà $M$, Pelletier S, Dorval M, Joly Y. Communication of genetic information in the palliative care context: ethical and legal issues. Med Law Int. 2018;18:219-40.

22. Reid GT, Walter FM, Brisbane JM, Emery JD. Family history questionnaires designed for clinical use: a systematic review. Public Health Genomics. 2009;12:73-83.

23. Cleophat JE, Nabi H, Pelletier S, Bouchard K, Dorval M. What characterizes cancer family history collection tools? A critical literature review. Curr Oncol. 2018;25:e335-50.

24. de Hoog CL, Portegijs PJ, Stoffers HE. Family history tools for primary care are not ready yet to be implemented. A systematic review. Eur J Gen Pract. 2014;20:125-33.

25. Qureshi N, Carroll JC, Wilson B, Santaguida P, Allanson J, Brouwers $\mathrm{M}$, et al. The current state of cancer family history collection tools in primary care: a systematic review. Genet Med. 2009;11:495-506. 